\title{
Simple Memristive SPICE Macro-Models and Reconfigurability in Filter and Antenna
}

\author{
Kai-da XU ${ }^{1}$, Xue-shi $L^{2}$, Ying-jiang GUO ${ }^{3}$, Qing-huo $L I U^{4}$ \\ ${ }^{1}$ Inst. of Electromagnetics and Acoustics \& Dept. of Electronic Science, Xiamen University, 361005 Xiamen, China \\ ${ }^{2}$ School of Automation, Guangdong University of Technology, 510006 Guangzhou, China \\ ${ }^{3}$ EHF Key Laboratory of Science, University of Electronic Science and Technology of China, 611731 Chengdu, China \\ ${ }^{4}$ Dept. of Electrical and Computer Engineering, Duke University, Durham, NC 27708, USA \\ lixueshi@gdut.edu.cn
}

Manuscript received April 29, 2016

\begin{abstract}
Simple current- and voltage-controlled memristive circuit macro-models using SPICE are proposed to capture the nonlinear hysteresis loop behaviors in this paper. Different current-voltage characteristics are investigated by applying sinusoidal-wave, triangular-wave and square-wave source, respectively. Furthermore, using finite-difference time-domain (FDTD) emulator incorporated with a SPICE circuit solver, the current- or voltagecontrolled memristive SPICE model is embedded into a planar microwave bandstop filter (BSF) and an ultrawideband (UWB) monopole antenna, which connects two ends of the half-wavelength open-loop resonator and two sides of the U-slot in the radiating patch, respectively. The reconfigurability of the $B S F$ and antenna notched band can be achieved by switching the states of the memristor.
\end{abstract}

\section{Keywords}

Bandstop filter, memristor, SPICE model, UWB antenna

\section{Introduction}

In 1971, the existence of the memristor as a new fourth electrical circuit element was predicted, and its formal mathematical definition was also given to connect the missing relation between the magnetic flux $(\phi)$ and electrical charge $(q)$ [1]. After a new nanoscale device based on crossbar architecture showing memristive behavior was fabricated in 2008 [2] whose schematic illustration is shown in Fig. 1(a), the memristor possesses significant potential for such next-generation nonvolatile memories [3]. Over the past several years, memristors have been applied in many fields, such as analog circuits [4], digital information progressing [5], neuromorphic [6], resistive random access memory (RRAM) [7], and microwave devices [8]. For all applications, a simple and straightforward theoretical model of the memristor is needed before physical realization. Till now, numerous memristive SPICE models have

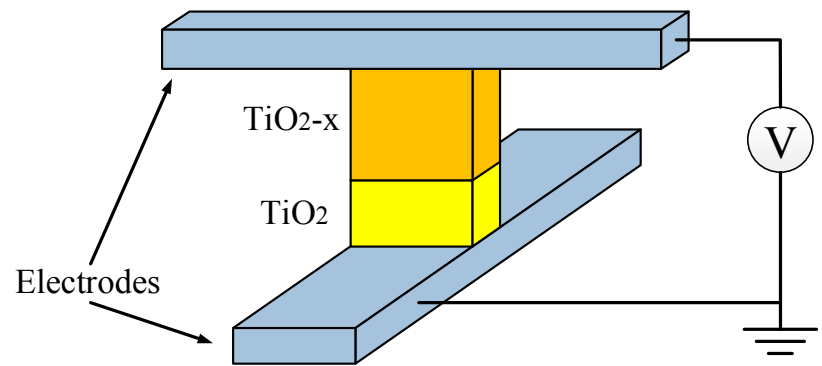

(a)

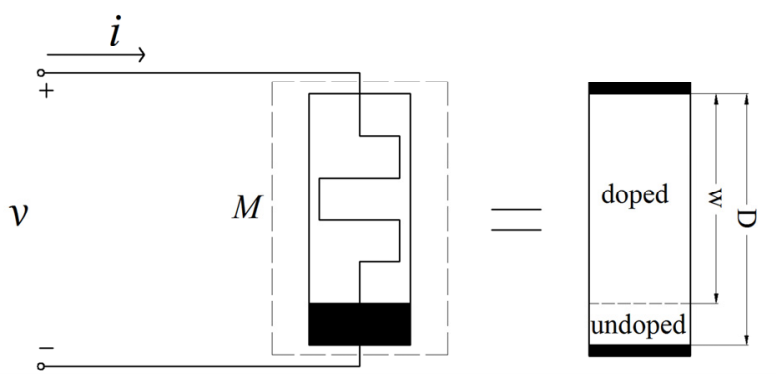

(b)

Fig. 1. (a) Schematic illustration of the memristor fabricated by Hewlett-Packard lab; (b) coupled variablememristor model.

been proposed in the literature [7-11] to mimic the hysteresis behaviors of the memristors, which are mostly based on the published mathematical equations in [2].

The relationship between voltage and current of the memristor in [2] was expressed by

$$
v(t)=\left[R_{\mathrm{off}}+\left(R_{\mathrm{on}}-R_{\mathrm{off}}\right) \cdot \bar{x}(t)\right] \cdot i(t)
$$

where the normalized state variable $\bar{x}(t)$ is the ratio between the doped region thickness $w$ and the whole thickness $D$ of the $\mathrm{TiO}_{2}$ memristor sandwiched region as seen in Fig. 1(b), $R_{\text {on }}$ and $R_{\text {off }}$ are the minimum and maximum achievable resistances of the memristor, respectively. The change rate of the state variable $\bar{x}(t)$ depends on the current through the memristor, which satisfies the following state equation,

$$
\frac{\mathrm{d} \bar{x}(t)}{\mathrm{d} t}=k i(t)
$$


On the other hand, switches or other tuning devices are commonly used to obtain switching or time-varying behaviors in microwave circuits. There are many ways to achieve switching properties, such as using micro- or nanoelectrical mechanical (MEM/NEM), electrical, and thermal technique. In terms of the memristor, the static nonvolatile resistance is controlled by the time-varying current or voltage. In this paper, simple current- and voltage-controlled memristive SPICE macro-models are presented, both of which can generate nonlinear hysteresis loop behaviors. To explore the promising reconfigurable functions of the memristor, the proposed current- or voltage-controlled memristive model is applied in a microwave BSF and an UWB antenna using finite-difference time-domain emulator incorporated with a nonlinear SPICE circuit solver.

\section{Proposed Memristive SPICE Macro-Models}

A symmetrical double hysteresis loop behavior can be produced with the following equation [12],

$$
y(t)=x(t)\left( \pm 1 \pm \frac{1}{T} \int_{0}^{t} x(\tau) \mathrm{d} \tau\right)
$$

where $x(t)$ is the normalized current/voltage input signal, $y(t)$ is the corresponding normalized voltage/current output signal, and $T$ is an integration time constant.

When $x(t)$ is represented by a current and $y(t)$ is represented by a voltage, a current-controlled memristive device model can be obtained. The equation can be changed as follows if we set $x(t)=i(t) / I_{\text {ref }}$, and $y(t)=v(t) /\left(I_{\text {ref }} R_{\mathrm{s}}\right)$,

$$
v(t)= \pm i(t) R_{\mathrm{s}} \pm \frac{i(t) R_{\mathrm{s}}}{T I_{\text {ref }}} \int_{0}^{t} i(\tau) \mathrm{d} \tau
$$

where $I_{\text {ref }}$ and $R_{\mathrm{s}}$ are an arbitrary reference current and an arbitrary resistance, respectively. Therefore, the memristance $R_{\mathrm{m}}=v(t) / i(t)$ can be given by

$$
R_{\mathrm{m}}= \pm R_{\mathrm{s}} \pm \frac{R_{\mathrm{s}}}{T I_{\text {ref }}} q(t) .
$$

$R_{\mathrm{m}}$ has four different possibilities, $(+,+),(+,-)$, $(-,+)$, and $(-,-)$, which represent incremental/decremental memristance and incremental/decremental negative memristance, respectively [12]. In SPICE, the positive or negative sign can be encoded by swapping nodes with each other. Figure 2 shows the proposed SPICE circuit macromodel of the current-controlled memristor, which only consists of two resistors $(R S$ and $R$ ), a capacitor $(C)$, a current dependent current source (CDCS), a voltage dependent voltage source (VDVS), an arbitrary behavioral voltage source (ABVS) and an arbitrary behavioral current source (ABCS). The integration time constant $T$ of (5) can be obtained by the product of $R$ and $C$ of the model, namely, $T=R C$.

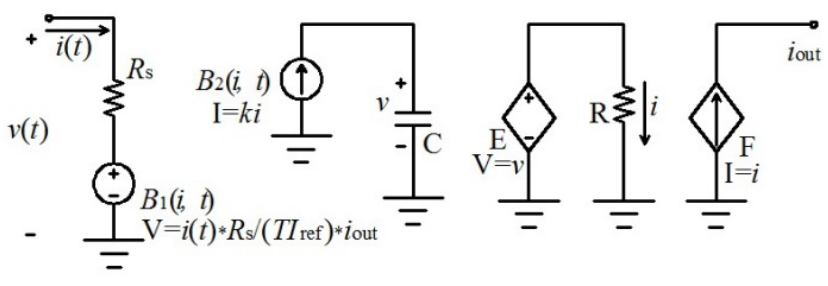

Fig. 2. Proposed current-controlled memristive model.

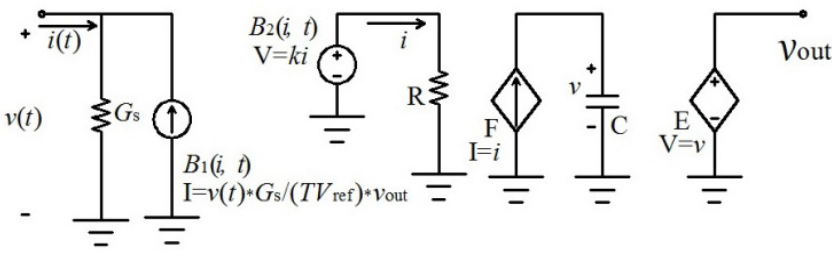

Fig. 3. Proposed voltage-controlled memristive model.

Similarly, when $y(t)$ is represented by a current and $x(t)$ is represented by a voltage, a voltage-controlled memristive device model is obtained. If we set $x(t)=v(t) / V_{\text {ref }}$, and $y(t)=i(t) /\left(V_{\mathrm{ref}} G_{\mathrm{s}}\right)$, Equation (3) can be expressed by

$$
i(t)= \pm G_{\mathrm{s}} v(t) \pm \frac{G_{\mathrm{s}} v(t)}{T V_{\text {ref }}} \int_{0}^{t} v(\tau) \mathrm{d} \tau
$$

where $V_{\text {ref }}$ and $G_{\mathrm{s}}$ are an arbitrary reference voltage and an arbitrary conductance, respectively. The conductance of the memristor is

$$
G_{\mathrm{m}}= \pm G_{\mathrm{s}} \pm \frac{G_{\mathrm{s}}}{T V_{\text {ref }}} \phi(t) .
$$

Therefore, the SPICE circuit macro-model of the voltagecontrolled memristor is proposed as shown in Fig. 3, which has the same components as those of the current-controlled memristive model. The integration time constant $T$ of (7) is still equal to the product of $R$ and $C$ of the model.

The hysteresis loop behavior can be obtained by either of the above two SPICE circuit models. Taking the current-controlled memristive SPICE model for instance, when $R C=1, R_{\mathrm{s}}=I_{\text {ref }}=1, i(t)=\cos (\omega t)$ and $\omega=1$, two cases $(+,+)$ and $(-,-)$ of (4) are plotted in Fig. 4(a), where they result in a positively inclined loop and a negatively inclined loop, respectively. This result is the same as that of [12]. For the $(+,+)$ and $(-,-)$ cases, the V-t and I-t

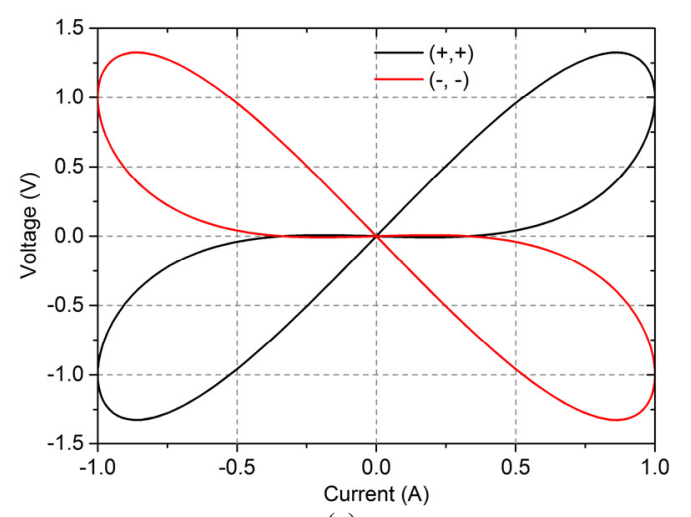

(a) 


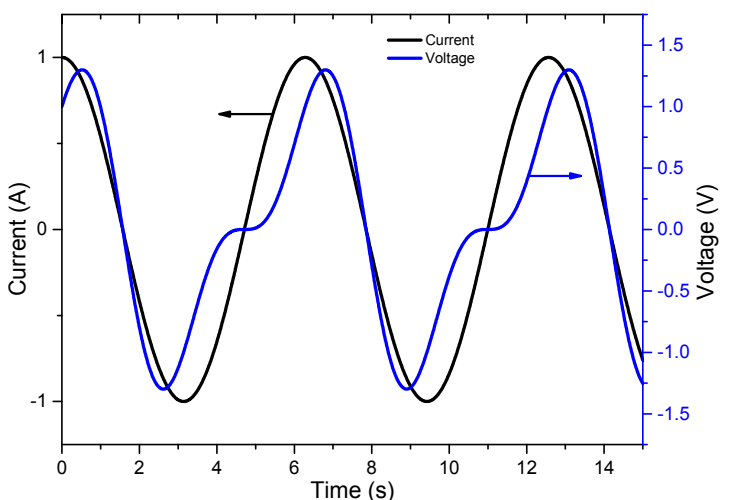

(b)

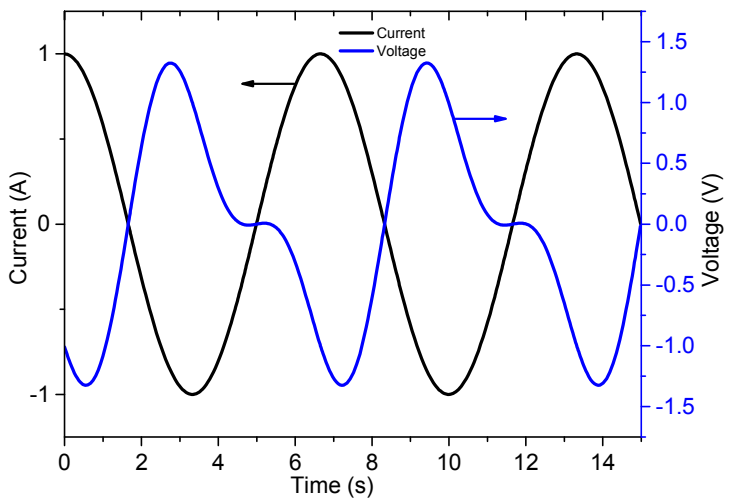

(c)

Fig. 4. (a) Double hysteresis loop behavior for $R C=1$ and $i(t)=\cos (t)$; V-t and I-t curves (b) for the $(+,+)$ case and (c) for the $(-,-)$ case when $i(t)=\cos (t)$.

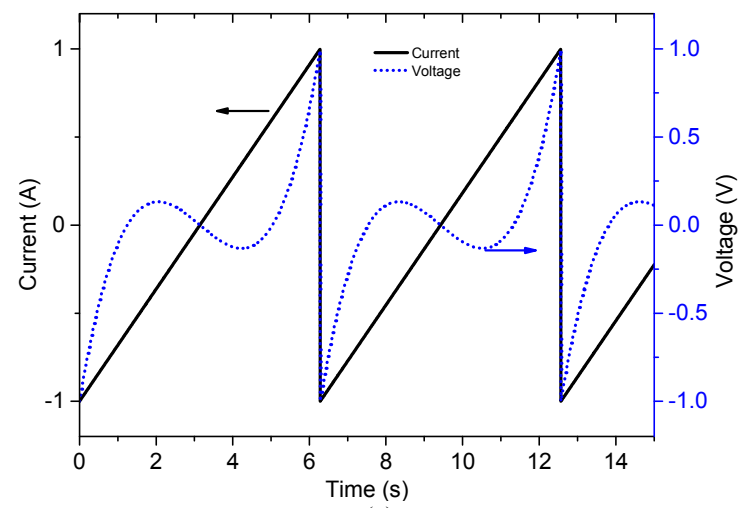

(a)

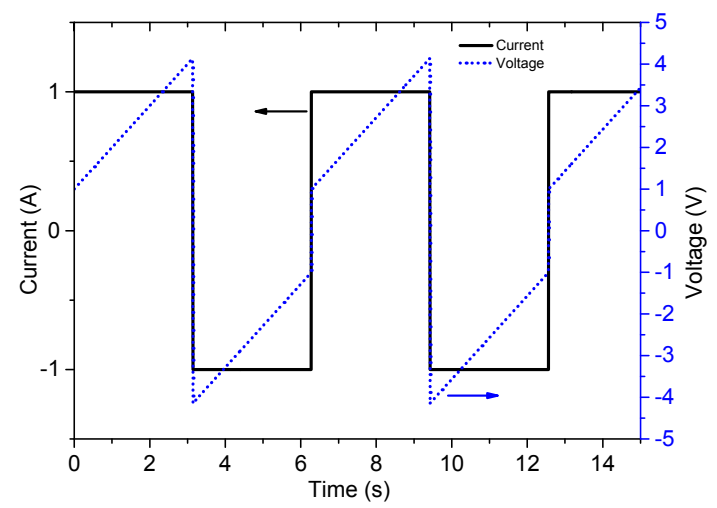

(b)

Fig. 5. V-t and I-t curves for the $(+,+)$ case driven by (a) triangular wave and (b) square wave currents.

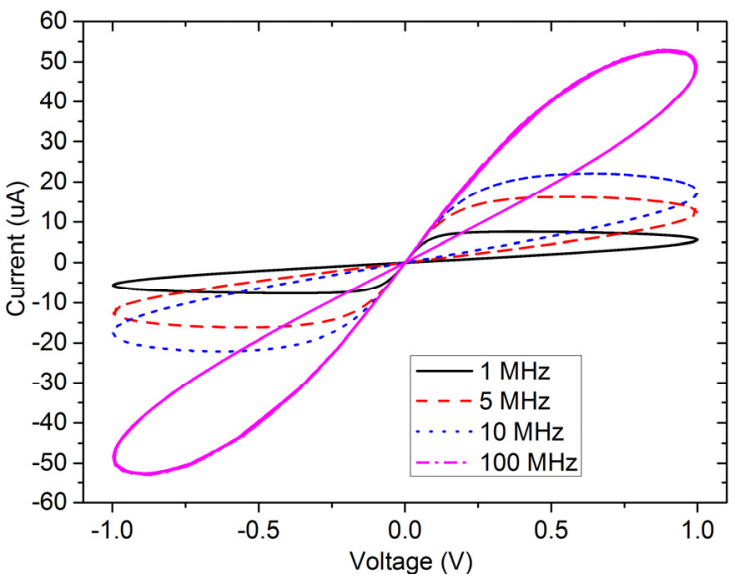

Fig. 6. $i-v$ characteristics of this memristive model driven by sinusoidal voltages at different frequencies with $I_{\mathrm{ref}}=1 \mathrm{~A}, T=1 \mathrm{~s}$, and $R_{\mathrm{s}}=10 \mathrm{k} \Omega$.

curves are plotted as shown in Fig. 4(b) and (c), respectively. Furthermore, when the triangular wave and square wave currents are as the inputs, the V-t and I-t curves for the $(+,+)$ case are illustrated in Fig. 5.

In addition, more behaviors with different currentvoltage characteristics of the proposed memristive SPICE model are investigated by applying sinusoidal source as shown in Fig. 6, where the simulated transient $i-v$ curves all have zero crossing properties at different frequencies.

\section{Applications in Microwave BSF and Antenna}

\subsection{Reconfigurable Microstrip BSF}

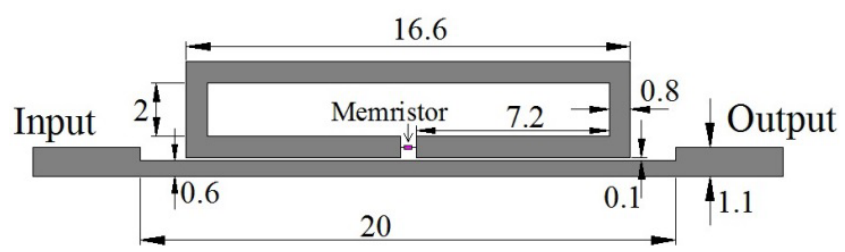

(a)

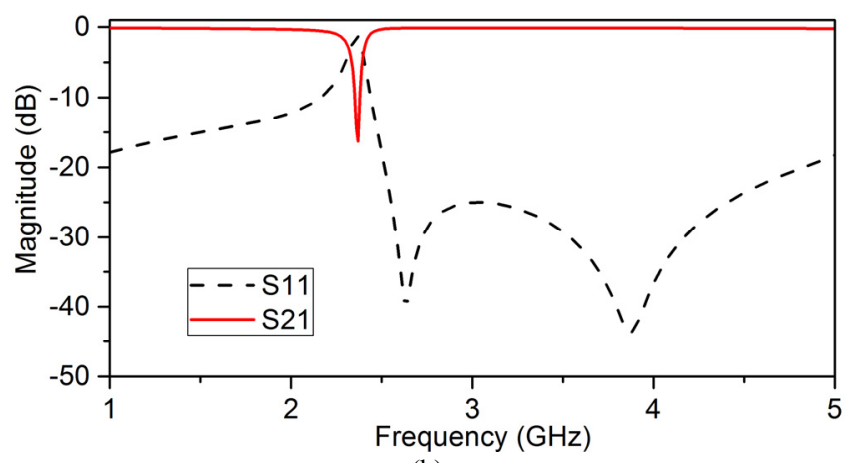

(b)

Fig. 7. (a) Layout of the microstrip BSF incorporated with a memristive SPICE model, where all dimensions are in $\mathrm{mm}$; (b) the scattering parameters of the BSF without memristor. 
Utilizing the above proposed memristive element model, a switchable microstrip BSF is designed as shown in Fig. 7(a). Without applying the memristor, this BSF designed on a substrate (with a relative dielectric constant of 3.5 and a thickness of $0.508 \mathrm{~mm}$ ) consists of a half-wavelength open-loop resonator and a transmission line connected to the input and output ports. Its scattering parameters can be seen in Fig. 7(b), where the insertion loss is $16.3 \mathrm{~dB}$ at $2.37 \mathrm{GHz}$.

The proposed memristive SPICE model is incorporated into this BSF connecting two ends of the open-loop resonator, and a reconfigurable BSF can be achieved by switching the states of the memristor. The whole model shown in Fig. 7(a) is simulated in a FDTD commercial simulation tool [13] integrated with the proposed memris-

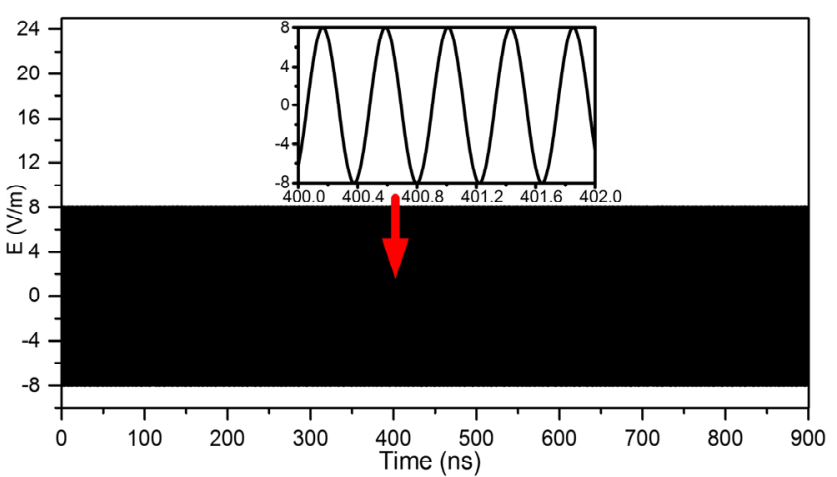

(a)

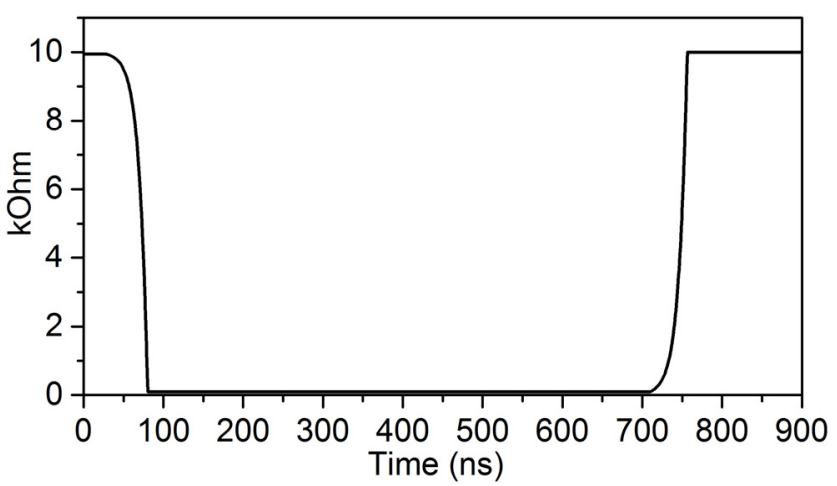

(b)

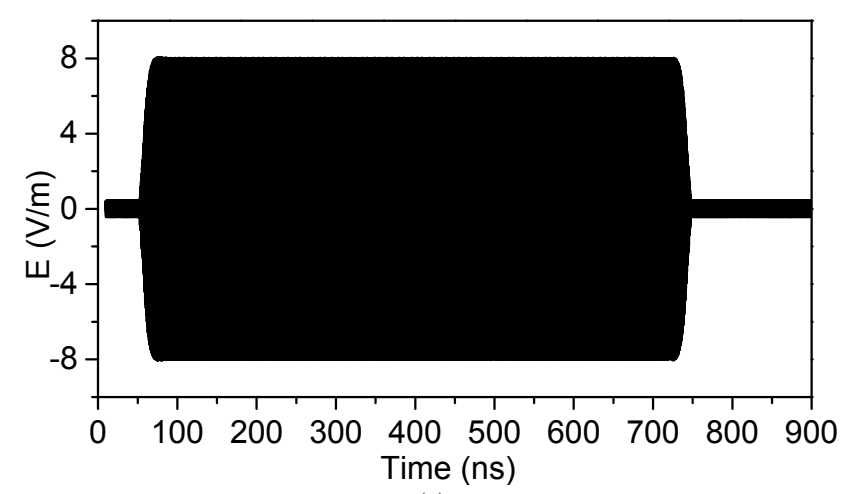

(c)

Fig. 8. (a) Transient incident electric field at the input port, (b) time-varying memristance, and (c) transient electric field at the output port. tive SPICE model. The applied voltage in series with the memristor is an effective electrical short circuit in microwave frequency range, resulting in no impact on the steady state performance of the filter. Figure 8(a) illustrates the transient incident electric field of $2.37-\mathrm{GHz}$ microwave signal. The inset in Fig. 8(a) shows the sinusoidal wave on a smaller time scale. The time-varying memristance controlled by the applied voltage is provided in Fig. 8(b), and meanwhile the instantaneous electric field at the output port can be obtained as shown in Fig. 8(c). At OFF-state $\left(R_{\mathrm{m}}=10 \mathrm{k} \Omega\right)$ of the memristor, the BSF keeps the original suppressed function at $2.37 \mathrm{GHz}$ to the incident field. Once the state is switched to $\mathrm{ON}\left(R_{\mathrm{m}}=50 \Omega\right)$, the electric field will not be attenuated and almost keep the same as that of the input signal.

\subsection{Reconfigurable UWB Monopole Antenna}

A planar UWB monopole antenna with U-shaped slot for band-notched operation is proposed as seen in Fig. 9 before the memristor is incorporated into this antenna, which consists of a microstrip-fed staircase-like taperedpatch monopole and a rectangular ground plane. The substrate RT/Duroid 5880 with a relative dielectric constant of 2.2 and a thickness of $1.575 \mathrm{~mm}$ is chosen for the design of this UWB antenna. The parameters $W t, L t, G_{1}, G_{2}, L_{\mathrm{S} 1}, L_{\mathrm{S} 2}$, $L_{1}, L_{2}, L_{\mathrm{g}}, W_{1}, W_{2}$, and $W_{\mathrm{S}}$ are optimized to ensure good performance, and the width of the $50 \Omega$ microstrip feed line $W$ equals to $4.9 \mathrm{~mm}$. The simulated voltage standing wave ratio (VSWR) results of the proposed UWB antenna with/without U-shaped slot including optimal parameters are shown in Fig. 10, where we can obtain that the Ushaped slot inserted in the radiating patch is to realize the frequency band-notched characteristics. The impedance bandwidth ranges from $2.7 \mathrm{GHz}$ to $11.2 \mathrm{GHz}(V S W R<2)$ before the slot is inserted. When the U-shaped slot is incorporated into this UWB antenna, the sharp notched frequency band of $3.8-4.1 \mathrm{GHz}$ is obtained. The slot only slightly interferes with the VSWR of the antenna except within the notched band. Actually, the total electrical length

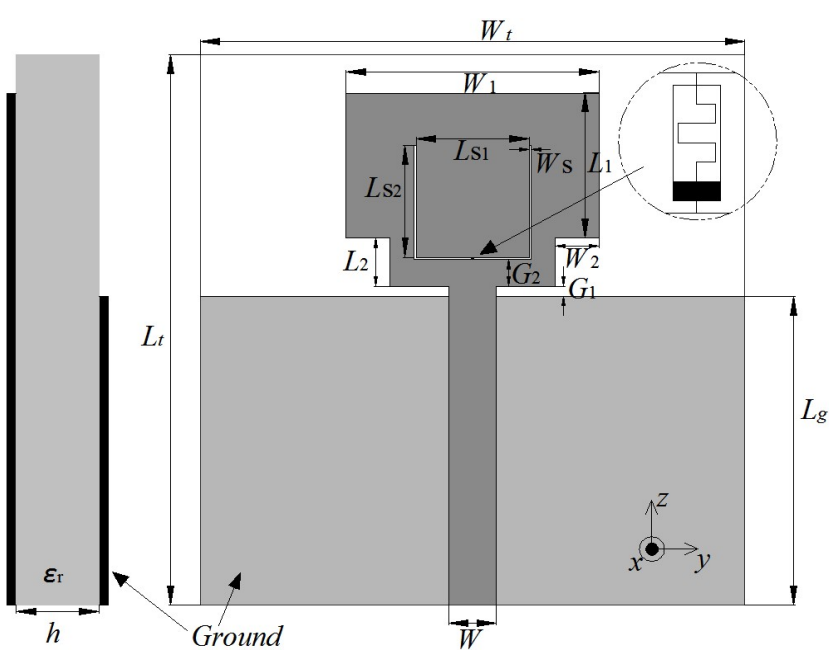

Fig. 9. Layout of the proposed antenna. 


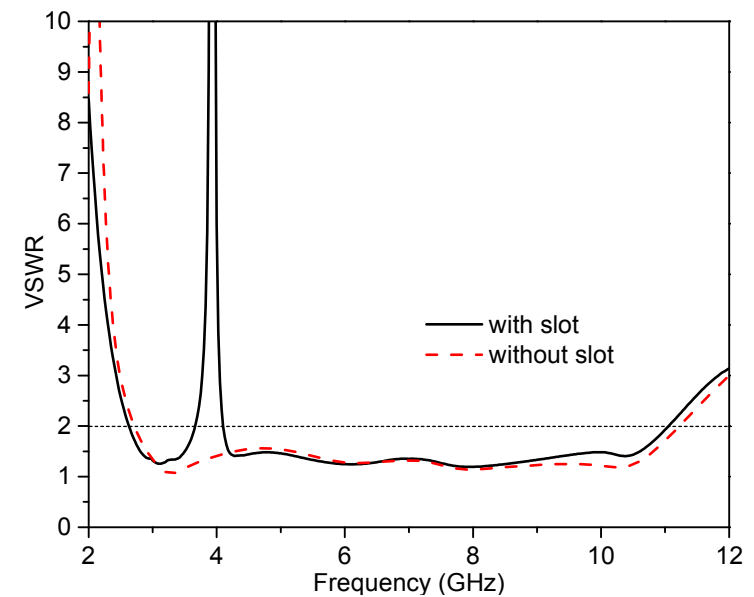

Fig. 10. The simulated VSWR results of the proposed UWB antenna with/without U-slot $(W t=36 \mathrm{~mm}, L t=57 \mathrm{~mm}$, $G_{1}=1 \mathrm{~mm}, G_{2}=2.8 \mathrm{~mm}, L_{\mathrm{S} 1}=L_{\mathrm{S} 2}=11.6 \mathrm{~mm}, L_{1}=15 \mathrm{~mm}$, $L_{2}=5 \mathrm{~mm}, L_{\mathrm{g}}=32 \mathrm{~mm}, W_{1}=26 \mathrm{~mm}, W_{2}=4.5 \mathrm{~mm}$, $\left.W_{\mathrm{S}}=0.2 \mathrm{~mm}\right)$.

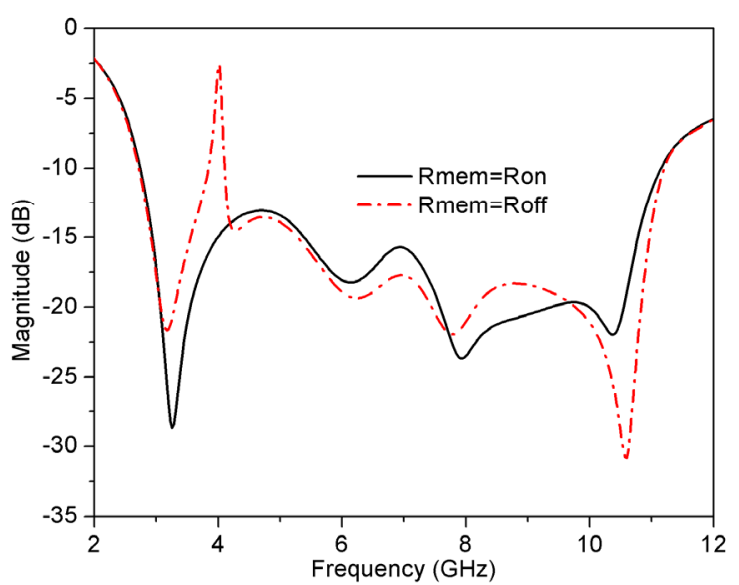

(a)

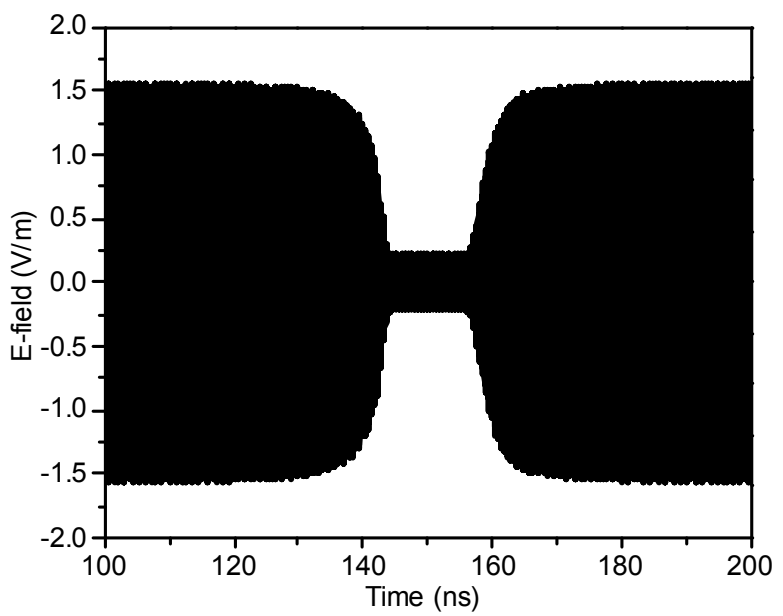

(b)

Fig. 11. (a) Simulated $S_{11}$ of the antenna when the state of the memristor is ON or OFF. (b) Transient E-field of the antenna when the memristor state is $\mathrm{ON} \rightarrow \mathrm{OFF} \rightarrow \mathrm{ON}$.

of the slot is about half guided wavelength at the center frequency of the notched band. Therefore, the notched frequency band can be moved by changing the values of $L_{\mathrm{S} 1}+2 L_{\mathrm{S} 2}$.
As illustrated in Fig. 9, the memristor using the proposed memristive SPICE model is embedded across the center of the U-shaped slot to achieve the reconfigurable notched band at 3.8-4.1 GHz. The nonvolatile memristor is used to activate and deactivate the slotting resonance by retaining either a high- or low-resistance state (see Fig. 11(a)). Due to the nonvolatile property of the memristor, when the applied voltage across the memristor is switched off, i.e., $v=0$ and $i=0$, at $t=t_{0}$, the high- or lowresistance state keeps unchanged for all $t>t_{0}$. We can keep the notched band of the antenna work or out of work all the time without a bias voltage. When the work state of the notched band needs to be changed, a relatively low bias voltage is only needed to switch the memristor from its high-resistance state to the low-resistance state as its counterpart. For instance, the transient electric field is observed by a receiver above the proposed antenna using an FDTD model. A 3.9-GHz sinusoidal wave signal whose frequency point is located in the notched band as a source signal could radiate from antenna when the memristor keeps ON state (100-140 ns or 160-200 ns) as shown in Fig. 11(b), nevertheless, it would be largely suppressed when the memristor switches from ON to OFF state (140-160 ns). Indeed, the memristor does not dissipate any power except during the only switching time intervals.

\section{Conclusion}

In this paper, using SPICE to emulate mathematical equations of the memristor, simple current- and voltagecontrolled memristive circuit macro-models are proposed. Different current-voltage characteristics are explored by applying sinusoidal-wave, triangular-wave and squarewave source across the memristor, respectively. Using FDTD technique, the memristive SPICE models embedded into a planar BSF and a monopole UWB antenna are conceived and simulated to achieve their reconfigurability. Having significant potential in the applications of $\mathrm{RF} / \mathrm{mi}$ crowaves, the memristor is greatly promising to be used in reconfigurable systems with low power dissipation and nanoscale size when it becomes a commercial product in the near future.

\section{Acknowledgments}

This work was supported in part by the Natural Science Foundation of Fujian Province of China (No. 2016J05164), and the National Natural Science Foundation of China (Nos. 61601390 and 61505035).

\section{References}

[1] CHUA, L. O. Memristor: The missing circuit element. IEEE Transactions on Circuit Theory, 1971, vol. 18, no. 5, p. 507-519. ISSN: 0018-9324. DOI: 10.1109/TCT.1971.1083337 
[2] STRUKOV, D. B., SNIDER, G. S., STEWART, D. R., WILLIAMS, S. R. The missing memristor found. Nature, 2008, vol. 453, no. 7191, p. 80-83. ISSN: 0028-0836. DOI: 10.1038 /nature 06932

[3] MEIJER, G. I. Who wins the nonvolatile memory race? Science, 2008, vol. 319 , p. 1625-1626. ISSN: 0036-8075. DOI: $10.1126 /$ science. 1153909

[4] SHIN, S., KIM, K., KANG, S. M. Memristor applications for programmable analog ICs. IEEE Transactions on Nanotechnology, 2010, vol. 10 , no. 2 , p. 266-274. ISSN: 1536-125X. DOI: 10.1109/TNANO.2009.2038610

[5] BORGHETTIL, J., SNIDER, G. S., KUEKES, P. J., YANG, J. J., STEWART, D. R., WILLIAMS, R. S. Memristive' switches enable 'stateful' logic operations via material implication. Nature Letters, 2010, vol. 464, no. 8, p. 873-876. ISSN: 0028-0836. DOI: 10.1038 /nature 08940

[6] KIM, H., SAH, M. P., YANG, C., ROSKA, T., CHUA, L. O. Neural synaptic weighting with a pulse-based memristor circuit. IEEE Transactions on Circuits and Systems I, 2012, vol. 59, no. 1, p. 148-158. ISSN: 1549-8328. DOI: 10.1109/TCSI.2011.2161360

[7] BIOLEK, Z., BIOLEK, D., BIOLKOVA, V. SPICE model of memristor with nonlinear dopant drift. Radioengineering, 2009, vol. 18, no. 2, p. 210-214. ISSN: 1210-2512.

[8] YAKOPCIC, C., TAHA, T. M., SUBRAMANYAM, G., PINO, R. E. Generalized memristive device SPICE model and its application in circuit design. IEEE Transactions on Computer-Aided Design of Integrated Circuits and Systems, 2013, vol. 32, no. 8, p. 1201 to 1214. ISSN: 0278-0070. DOI: 10.1109/TCAD.2013.2252057

[9] XU, K. D., ZHANG, Y. H., WANG, L., YUAN, M. Q., FAN, Y., JOINES, W. T., LIU, Q. H. Two memristor SPICE models and their applications in microwave devices. IEEE Transactions on Nanotechnology, 2014, vol. 13, no. 3, p. 607-616. ISSN: 1536125X. DOI: $10.1109 /$ TNANO.2014.2314126

[10] RAK, A., CSEREY, G. Macromodeling of the memristor in SPICE. IEEE Transactions on Computer-Aided Design of Integrated Circuits and Systems, 2010, vol. 29, no. 4, p. 632-636. ISSN: 0278-0070. DOI: 10.1109/TCAD.2010.2042900

[11] SHIN, S., KIM, K., KANG, S. M. Compact models for memristors based on charge-flux constitutive relationships. IEEE Transactions on Computer-Aided Design of Integrated Circuits and Systems, 2010, vol. 29 , no. 4, p. 590-598. ISSN: 0278-0070. DOI: 10.1109/TCAD.2010.2042891

[12] ELWAKIL, A. S., FOUDA, M. E., RADWAN, A. G. A simple model of double-loop hysteresis behavior in memristive elements. IEEE Transactions on Circuits and Systems II, Express Briefs, 2013, vol. 60, no. 8, p. 487-491. ISSN: 1549-7747. DOI: 10.1109/TCSII.2013.2268376

[13] WAVE COMPUTATION TECHNOLOGIES, Inc. (WCT), Wavenology EM User's Manual, 2012.

\section{About the Authors ...}

Kai-da XU was born in Zhejiang, China. He received the B.S. and Ph.D. degrees in Electromagnetic Field and Microwave Technology from the University of Electronic Science and Technology of China (UESTC), Chengdu, China, in 2009 and 2015, respectively. From September 2012 to August 2014, he was a Visiting Researcher in the Dept. of Electrical and Computer Engineering, Duke University, Durham, NC, under the financial support from the China Scholarship Council (CSC). He received the UESTC
Outstanding Graduate Awards in 2009 and 2015, respectively. He was the recipient of the National Graduate Student Scholarship in 2012, 2013, and 2014 from the Ministry of Education, China. He is now an Assistant Professor with the Inst. of Electromagnetics and Acoustics, and the Dept. of Electronic Science, Xiamen University, Xiamen, China. He has published over 40 papers in peer-reviewed journals and conference proceedings. Since 2014, he has served as a reviewer for some journals including IEEE Transactions on Microwave Theory and Techniques, IEEE Transactions on Electron Devices, IEEE Transactions on Computer-Aided Design of Integrated Circuits and Systems, IEEE Transactions on Applied Superconductivity, ACES Journal, PIER, JEMWA and so on. His research interests include $\mathrm{RF} /$ microwave and $\mathrm{mm}$-wave circuits, antennas, and nanoscale memristors.

Xue-shi LI (corresponding author) was born in Sichuan, China. He received the B.E. degree in Electronic Engineering from Hohai University (HHU), Nanjing, China in 2004, the M.E. degree in Circuit and System from the South China Normal University (SCNU), Guangzhou, China in 2007, and the Ph.D. degree in Electromagnetic Field and Microwave Technology from the South China University of Technology (SCUT), Guangzhou, China in 2010. During October 2011 to October 2012, he was a visiting scholar in the Dept. of Electrical and Computer Engineering, Duke University, Durham, NC. He is now a lecturer with the School of Automation, Guangdong University of Technology, Guangzhou, China. He has published over 20 papers in peer-reviewed journals and conference proceedings. He has filed/granted a number of China patents in plasma antennas and metamaterial antennas. His research interests include computational electromagnetics, surface plasmon polaritons and metamaterial antennas.

Ying-jiang GUO was born in Sichuan, China. He received B.E. degree in Electronic Engineering from the Sichuan University (SCU), Chengdu, China in 2008, and received M.E. in Electromagnetic Field and Microwave Technology from the University of Electronic Science and Technology of China (UESTC), Chengdu, China in 2011, where he is currently working toward the Ph.D. degree in Electromagnetic Field and Microwave Technology. From 2011 to 2013, he was with the Chengdu Research Institute of Huawei Technology Ltd., where he was involved in the pre-research of ultra-wideband power amplifier, high frequency clock for $\mathrm{AD}$ and $5 \mathrm{G}$ communication prototype building. From 2013 to 2014, he was with the Sichuan Normal University, where he was a lecturer. $\mathrm{He}$ has filed/granted a number of China patents in microwave circuit and internet of vehicle. His research interests include $\mathrm{RF} /$ microwave/mm-wave transceiver design and monolithic-microwave integrated circuit applications.

Qing-huo LIU received the B.S. and M.S. degrees in Physics from Xiamen University, Fujian Province, China, in 1983 and 1986, respectively, and the Ph.D. degree in Electrical Engineering from the University of Illinois at Urbana-Champaign, in 1989. He was with the Electro- 
magnetics Laboratory, University of Illinois at UrbanaChampaign, as a Postdoctoral Research Associate from January 1989 to February 1990. He was a Research Scientist and Program Leader with Schlumberger-Doll Research, Ridgefield, CT, from 1990 to 1995. From 1996 to May 1999, he was an Associate Professor with the New Mexico State University. Since June 1999, he had been with Duke University, Durham, NC, where he is now a Professor of Electrical and Computer Engineering. He is also a guest Chair Professor at Xiamen University, Fujian, China. His research interests include computational electromagnetics and acoustics, inverse problems, geophysical subsurface sensing, biomedical imaging, electronic packaging, and the simulation of photonic and nano-devices. He has published more than 550 papers in refereed journals and conference proceedings. He is a Fellow of the Acoustical Society of America, a member of Phi Kappa Phi, Tau Beta Pi, a full member of the U.S. National Committee of URSI Commissions B and F. Currently he serves as the Deputy Editor in Chief of Progress in Electromagnetics Research, an Associate Editor for IEEE Transactions on Geoscience and Remote Sensing, and an Editor for the Journal of Computational Acoustics. He received the 1996 Presidential Early Career Award for Scientists and Engineers (PECASE) from the White House, the 1996 Early Career Research Award from the Environmental Protection Agency, and the 1997 CAREER Award from the National Science Foundation. 